\title{
Safety of a D-dimer based strategy and repeated ultrasonography did not differ in DVT and normal proximal vein ultrasonography
}

Kearon C, Ginsberg JS, Douketis J, et al. A randomized trial of diagnostic strategies after normal proximal vein ultrasonography for suspected deep venous thrombosis: D-dimer testing compared with repeated ultrasonography. Ann Intern Med 2005; 142:490-6.

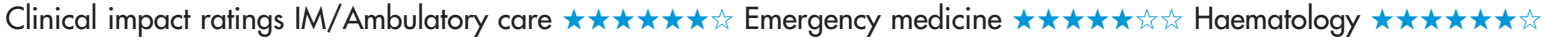

In patients with suspected deep venous thrombosis (DVT) and negative results on proximal vein ultrasonography, how does a D-dimer-based management strategy that minimises additional assessments compare with routine repeated ultrasonography?

\section{METHODS}

ட

Design: randomised controlled trial.

Allocation: concealed.

Blinding: blinded (outcome assessors).*

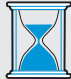

Follow up period: 6 months.

Setting: thrombosis services of 4 university hospitals in Hamilton Ontario, Canada.

Patients: 810 patients (mean age $59.5 \mathrm{y}, 62 \%$ women) with a suspected first episode of DVT and negative results on proximal vein ultrasonography who were referred by primary care and hospital-based physicians to a thrombosis outpatient service. Exclusion criteria included life expectancy $<6$ months, contraindication to venography, use of full dose heparin for $>48$ hours, use of long term warfarin therapy, symptoms of pulmonary

RX

Intervention: erythrocyte agglutination D-dimer testing with no further testing after a negative result and venography affer a positive result (D-dimer strategy, $n=408$ ); or routine repeated ultrasonography at 1 week (repeated ultrasonography, $n=402$ ). Patients in the D-dimer strategy group who had DVT shown on venography were treated with anticoagulants for $\geqslant 3$ months. Patients in the repeated ultrasonography group only received anticoagulant therapy if the second ultrasound was abnormal. and safety (assessed by rate of symptomatic venous thromboembolism in patients without DVT on initial testing at $6 \mathrm{mo}$ ).

*See glossary. embolism, and pregnancy.

Outcomes: initial diagnosis and treatment of symptomatic DVT

$\square$ Patient follow up: $96 \%$

\section{MAIN RESULTS}

$4.7 \%$ of patients in the D-dimer strategy group and $0.7 \%$ of patients in the repeated ultrasonography group received a diagnosis of DVT at initial testing and treatment $(\mathrm{p}<0.001)$. In patients without DVT on initial testing, groups did not differ for the rate of DVT at 6 months (table).

\section{CONCLUSION}

In patients with suspected deep venous thrombosis and negative results on proximal vein ultrasonography, the safety of a D-dimer based management strategy that minimises additional assessments did not differ from that of routine repeated ultrasonography.

\section{Commentary}

$\mathrm{K}$ earon et al report on a new strategy to refute the diagnosis of symptomatic DVT efficiently in a single visit. This study did not use a pre-test probability score for clinical decision making, but included this information descriptively. Relatively few patients in the D-dimer strategy group were in the moderate $(30 \%)$ or high $(5 \%)$ pre-test probability groups.

Posthoc analysis by pre-test probability score shows events rates of $4.5 \%$ (estimated $95 \% \mathrm{Cl} 0.24$ to $25 \%$ ) among high probability patients and $2.4 \%$ (estimated $\mathrm{Cl} 0.63$ to $7.5 \%$ ) among moderate probability patients. Interestingly, half of these patients had events following a single negative compression ultrasound and a negative D-dimer, a strategy that has been validated previously. ${ }^{12}$ As can be appreciated by the wide Cls, limited conclusions can be reached by analysing subgroups in a study not designed for such analysis. As summarised by Hulley et al, ${ }^{3}$ this study is useful to the extent that it yields valid inferences, first about events that happened in the study sample ("internal validity"), and then about generalising results to patients outside the study ("external validity"). This well designed and executed trial possesses internal validity; the conclusion that it is safe to withhold anticoagulation based on the interventions tested is valid for the population studied. Clinicians can always ask whether the patient whom they are evaluating was represented in the population studied, and hence whether or not the results provide information applicable to the evaluation of their patient ("external validity"). When confronted with a patient with known pre-test probability that is moderate or high, clinicians should apply the conclusions of this study with great caution. A prospective study testing this strategy on such patients is needed.

Scott M Stevens, MD, C Gregory Elliott, MD LDS Hospital and University of Utah School of Medicine Salt Lake City, Utah, USA

For correspondence: Dr $\mathrm{C}$ Kearon, McMaster University and the Henderson Research Centre, Hamilton, Ontario, Canada

Sources of funding: National Health Research Development Program of Health Canada; Heart and Stroke Foundation of Canada; Canadian Institutes of Health Research.

D-dimer strategy $v$ repeated US in suspected DVT with negative results on proximal vein US and no DVT on initial testing*

\begin{tabular}{lllll}
\hline Outcome at 6 months & D-dimer & Repeated US & RRI (95\% Cl) & NNH \\
\hline Symptomatic DVT & $2.1 \%$ & $1.3 \%$ & $64 \%(-43$ to 372) & Not significant \\
\hline
\end{tabular}

*DVT = deep venous thrombosis; US = ultrasonography. Other abbreviations defined in glossary; RRI, NNH, and Cl calculated from data in article. 